\title{
Development of a borehole stress meter for studying earthquake predictions and rock mechanics, and stress seismograms of the 2011 Tohoku earthquake ( $M$ 9.0)
}

\author{
Hiroshi Ishii ${ }^{*}$ and Yasuhiro Asai
}

\begin{abstract}
Although precursory signs of an earthquake can occur before the event, it is difficult to observe such signs with precision, especially on earth's surface where artificial noise and other factors complicate signal detection. One possible solution to this problem is to install monitoring instruments into the deep bedrock where earthquakes are likely to begin. When evaluating earthquake occurrence, it is necessary to elucidate the processes of stress accumulation in a medium and then release as a fault (crack) is generated, and to do so, the stress must be observed continuously. However, continuous observations of stress have not been implemented yet for earthquake monitoring programs. Strain is a secondary physical quantity whose variation varies depending on the elastic coefficient of the medium, and it can yield potentially valuable information as well. This article describes the development of a borehole stress meter that is capable of recording both continuous stress and strain at a depth of about $1 \mathrm{~km}$. Specifically, this paper introduces the design principles of the stress meter as well as its actual structure. It also describes a newly developed calibration procedure and the results obtained to date for stress and strain studies of deep boreholes at three locations in Japan. To show examples of the observations, records of stress seismic waveforms generated by the 2011 Tohoku earthquake (M 9.0) are presented. The results demonstrate that the stress meter data have sufficient precision and reliability.
\end{abstract}

Keywords: Development of borehole stress meter; Continuous observations of stress and strain; Earthquake prediction; Calibration system; 2011 Tohoku earthquake (M 9.0); Stress-water pressure seismograms

\section{Background}

Observing precursory phenomena of earthquakes is difficult; however, a case in which precursory fluctuations in strain and inclination were actually observed does exist, although the observations were discovered after the earthquake. This case was related to the swarm earthquakes that often occur in the Izu Peninsula, which is located approximately $100 \mathrm{~km}$ southwest of Tokyo (Ishii et al. 1998; Okada et al. 2000; Ishii et al. 2002). The eastern part of the Izu Peninsula is being uplifted at an annual rate of about $2 \mathrm{~cm} /$ year. One of the borehole meters that we developed is installed in a deep borehole in the eastern Izu Peninsula. This meter

\footnotetext{
* Correspondence: hirohiroishii@gmail.com

Tono Research Institute of Earthquake Science (TRIES), Association for the Development of Earthquake Prediction (ADEP), 1-63 Yamanouchi Akiyo-cho, Mizunami City, Gifu Prefecture 509-6132, Japan
}

recorded precursory inclination and strain fluctuations before the occurrence of the swarm earthquakes. It was discovered that the precursory phenomena were indicative of tectonic effects and that multi-component observations in deep boreholes is an effective technique for evaluating earthquake occurrences.

Several types of strain observation meters are used in boreholes. To date, volumetric strain meters (Sacks et al. 1971) have been installed at more than 30 locations in the Kanto and Tokai regions by the Japan Meteorological Agency. This type of meter can be used to observe changes in volumetric strain only. The principle of the meter is to convert the movement of silicone oil sealed inside a cylindrical vessel into changes in strain. Sakata (1981) developed a three-component strain meter by applying a Sacks-like principle. These meters are sensitive 
to temperature changes as the silicone oil is sealed inside the container. Gladwin (1984) developed a three-component strain meter based on the principle of measuring changes in the diameter of a cylindrical vessel by using a capacitance conversion sensor.

Ishii et al. (1997a, 2002) developed a strain meter that had improved sensitivity as a result of mechanically expanding the deformation of the cylindrical vessel. We have developed a comprehensive borehole crustal activity observation device (comprehensive observation device) equipped with strain meters and also tiltmeters, seismometers, and other components that are all co-located in one borehole container. We developed this comprehensive device because we think it is important to obtain as much information as possible to further advance the field of earthquake prediction research.

The comprehensive observation device developed by our institute has been adopted for use by the Japan Meteorological Agency, the National Institute of Advanced Industrial Science and Technology, and others. Such devices are installed in many deep boreholes and are being used to accumulate data. Strain was being analyzed from observational records of the strain meter at the Byobusan borehole observation point (depth of 1,020 m), which is managed by the Tono Research Institute of Earthquake Science when the Kii Peninsula earthquake ( $M 7.1$ and $M$ 7.4) occurred in the southeastern offshore on 5 September 2004 (Okubo et al. 2004; Asai et al. 2005) and when the Sumatra-Andaman earthquake ( $M$ 9.1) occurred on 26 December 2004 (Okubo et al. 2005). Crustal deformations concurrent with deep low-frequency tremors have also been observed with the strain meters installed in the deep boreholes, and additional strain analysis studies are being conducted (Itaba et al. 2010).

The Tohoku earthquake ( $M$ 9.0) occurred on 11 March 2011, and there are a number of excellent studies about the earthquake (e.g., Nishimura et al. 2011; Yoshida et al. 2011). Outcomes regarding strain seismic motions and crustal deformations concurrent with the 2011 Tohoku earthquake ( $M$ 9.0) have been delivered from observational data of meters installed at many deep borehole observation points (Itaba et al. 2011). Results have also been reported based on observations from stress meters installed at deep borehole observation points (Ishii and Asai 2011).

However, if we want to study earthquake occurrences comprehensively, it would be best to examine the processes of stress accumulation and subsequent release as a fault is generated. It is assumed that earthquakes occur when the stress applied at the fault surface exceeds frictional strength. Thus, the final objective in earthquake prediction research is to monitor the stress distribution and fault strength in earthquake-prone areas over time and space (Ishii et al. 1997b; Iio 2009).
At present, stress measurements are conducted using hydraulic fracturing, stress release methods, and so forth. However, these can only be used to determine the stress value at the point of measurement and cannot be used for continuous observations (Sano 2005). Consequently, we developed a meter capable of continuously observing the stress change. This stress meter can observe strain as well as stress. This article describes the stress meter system and reports case examples of observational results.

\section{Methods}

\section{Principle and design of the stress meter}

At present, the borehole-type strain meters developed by Ishii et al. (2002) are installed for earthquake prediction research at many deep borehole observation sites in Japan. Borehole-type strain meters detect fluctuations in the strain of bedrock (extension and contraction of the bedrock) continuously over long periods of time after they are set within a borehole and integrated with the bedrock by expansible grout with an expansion coefficient of about $0.15 \%$. With conventional technology, strain in the bedrock is detected based on the deformation in the cylindrical case.

The most important factor to be measured when observing the elastoplastic deformation of bedrock for earthquake prediction research is stress, which is directly related to the occurrence of earthquakes. However, conventional technology detects strain in bedrock, and the only way to obtain the continuous changes in stress is to calculate these based on continuous observations of bedrock strain through complex operations using the pre-measured elastic coefficient for the bedrock. That is, stress in bedrock can be obtained only indirectly using conventional technology. To date, no equipment that can directly and continuously detect both stress and strain in bedrock independently over a long period of time has been developed.

The stress meter that we have developed is capable of detecting both stress and strain. It is comprised of a pillar-shaped case and a pressure-receiving system with two pressure-receiving surfaces that are subjected to stress from the bedrock. Figure 1 shows a comparison of the outside view between a conventional strain meter and the newly developed stress meter. The stress meter has two pressure-receiving surfaces that are exposed on the outer wall of the case, but the strain meter has only the cylindrical case.

We next compare the internal structures of the stress meter and strain meter. Figure 2 shows front views of the internal parts of the two meters. The strain meter (on the right) has a frame, magnifying device (Ishii et al. 2002), displacement sensor, and so forth inside the cylindrical case. Deformation in the cylindrical case causes 


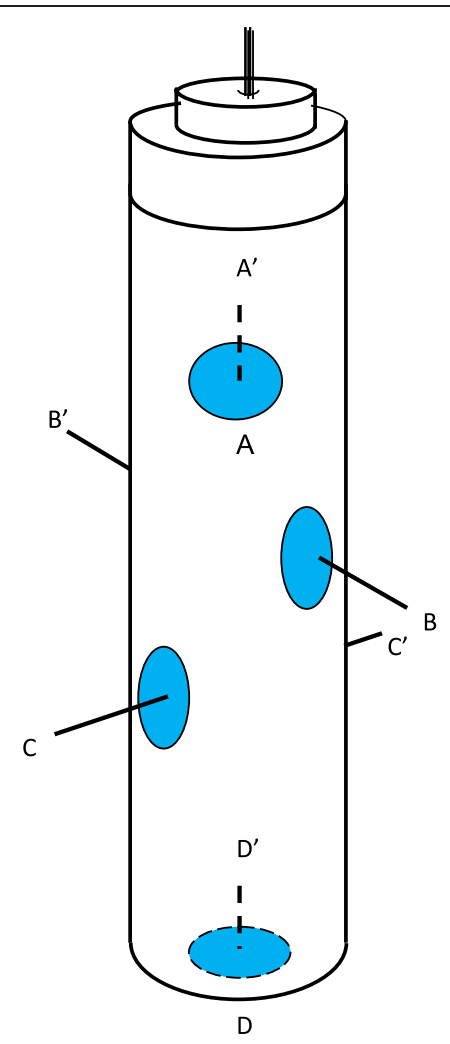

Stress meter

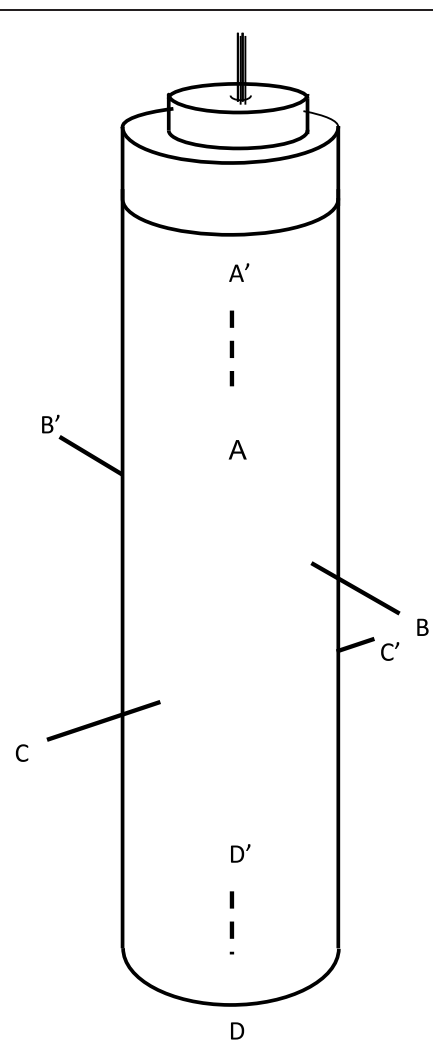

Strain meter

Figure 1 Schematic depiction of the stress meter and strain meter from the outside view. The blue ellipses are the pressure-receiving surfaces that are explained in the text.

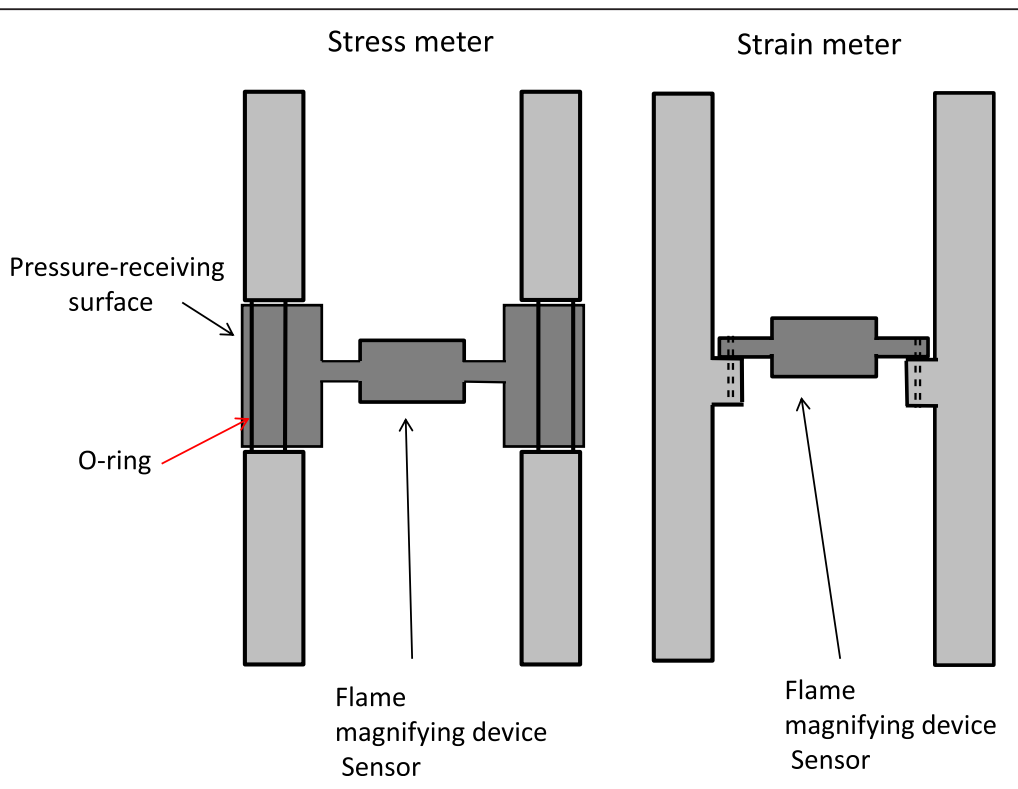

Figure 2 Front view of the schematic depiction of the stress meter and strain meter for one component. 
deformation in the frame, which is increased by the magnifying device and converted to a voltage by the displacement sensor. The stress detection part of the stress meter (on the left) is comprised of two pressure-receiving surfaces, a frame, a magnifying device, and a displacement sensor (pressure receiving system). This pressure-receiving system is on the axis orthogonal to the case axis and is exposed at the outer wall. An O-ring is installed in the space next to the case and is thus movable in the axial direction without a mechanical connection. The device also incorporates a displacement-detection sensor to detect the stress and strain in the bedrock based on the displacement between the two pressure-receiving surfaces.

Figure 3 compares the top views of the internal parts. The strain meter (right) has a frame, magnifying device, displacement sensor, and so forth inside its cylindrical case. The stress meter (left) has a pressure-receiving system that is comprised of two pressure-receiving surfaces that are located on the axis orthogonal to the case axis and exposed at the outer wall of the case.

Although the case deforms in response to stress or strain from the bedrock, the displacement of the pressurereceiving surface is not affected by the deformation of the case because the pressure-receiving surface material is not mechanically connected to the case, which makes it possible to detect both stress and strain in the bedrock accurately. The two pressure-receiving surfaces are connected to the ring-shaped connection component, which undergoes elastic deformation when subjected to stress. When the stress applied to the pressure-receiving surfaces is removed, the connection component is restored to its original shape and the displacement between the two pressure-receiving surfaces also returns to the value that existed before the surfaces were subjected to stress from the bedrock. The pressure-receiving surface material is installed at several points in different directions against the case, so it is possible to detect stress from the bedrock or strain in the bedrock applied in any axial direction independently.

The stress applied to the two pressure-receiving surfaces from the bedrock is magnified by the stress magnification mechanism, which successively magnifies using the principle of leverage, and the displacement at the final end of the lever is detected by the displacement detection sensor (Ishii et al. 2002). This magnification mechanism is usually used with a magnification rate of approximately 40 times. Thus, even small displacements between the two pressure-receiving surfaces are magnified by this mechanism, and low levels of stress applied to the pressure-receiving surfaces from the bedrock or strain in the bedrock can be detected.

In normal strain meters, the overall deformation of the case becomes correlated and it may be difficult to accurately and independently detect strain in the bedrock applied against the case from a specified direction.

\section{The developed observation instrument}

We developed a stress meter based on the previously described principles. Figure 4 shows a photograph of the construction of the one-component stress meter. The photograph shows the one-component cylindrical vessel, pressure-receiving system, pressure-receiving surface, and mechanical magnifying system. The detection unit was manufactured as an integrated type, so it delivers an elastic deformation response in response to an increase or decrease in applied pressure. The magnifying system applies the principle of leverage for magnifying the applied displacement. It is normally set to a magnification rate of approximately 40 times, and it is usually installed inside the detection unit. The pressure-receiving surfaces are installed on both sides of the detection unit, and the

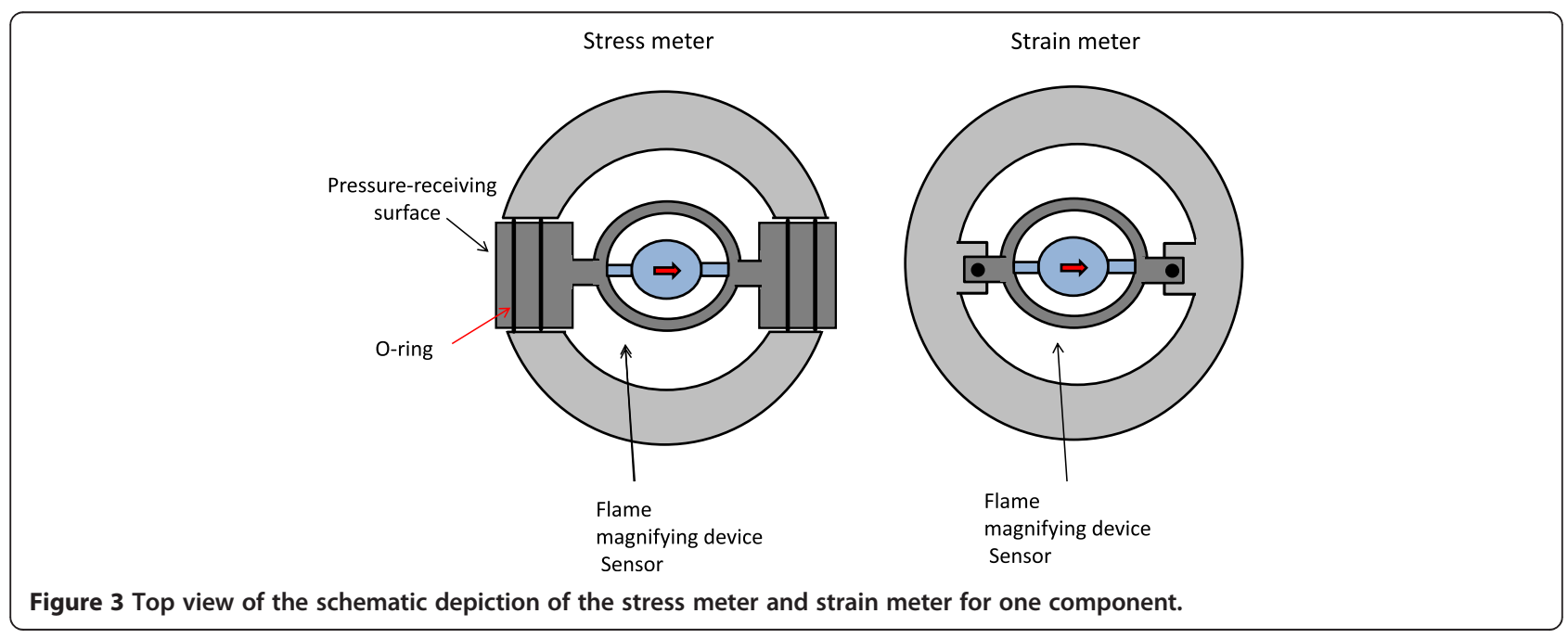




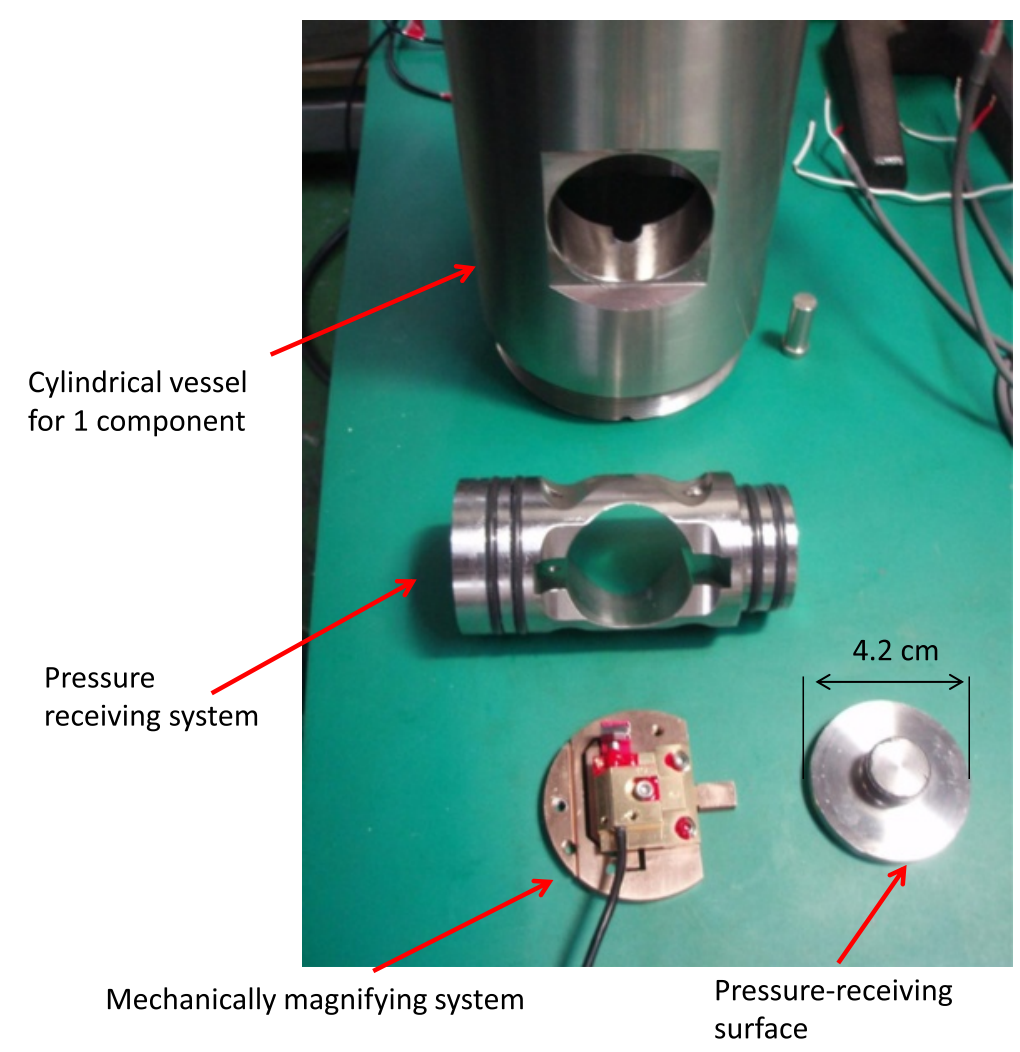

Figure 4 Photograph showing the structure of the stress meter for one component.

detection unit is incorporated into the cylindrical vessel. Figure 5 shows the vessel with the detection unit installed. The detection unit and vessel are in contact with each other via only an O-ring, and there is no mechanical connection. Therefore, deformation of the vessel does not affect the detection unit, and only the stress applied to the pressure-receiving surfaces is detected. Figure 6 is a photograph of the detection unit showing the horizontal components incorporated in the vessel. Half of the vessel is cut out to show the interior. The pressure-receiving surfaces, O-ring, and magnifying system can be observed visually. Figure 7 shows a photograph of a detection unit for a slant component incorporated in the vessel, again with half of the vessel cut out to reveal the interior. The stress meter usually incorporates four horizontal components, two slant components, and the vertical component.

For each component of the stress meter, a mechanism capable of mechanical zero adjustment is installed as necessary. This mechanism adjusts by manually operating a built-in motor, and it can be used to reset the meter in case of a large secular variation.

The stress meter is usually used as a component incorporated in the comprehensive crustal activity observation device that we have developed. Figure 8 shows a comprehensive observation device installed at the Toshinomori (TOS) borehole station (depth of $512 \mathrm{~m}$ ), which is one of our borehole observation stations. The TOS station $\left(35.335^{\circ} \mathrm{N} ; 137.218^{\circ} \mathrm{E}\right)$ is located in the Gifu Prefecture in central Japan. The stress meter at TOS is incorporated along with tilt meters, seismometers, a thermometer, and magnetometers. A comprehensive observation report on the device will be made separately. To observe stress and strain using the meters, the device must be integrated with the bedrock. Therefore, the comprehensive crustal activity observation device incorporating the stress meter and other components is fixed inside a deep borehole with expansible grout. Thus, the meters cannot be easily removed once buried and installed. The greatest cause of meter malfunction is lightning strikes, so the meters are equipped with various lightning resistance measures.

\section{Examination of stress meter sensitivity}

The stress meter can observe both stress and strain. Therefore, sensitivity to both stress and strain must be examined. A new examination device was developed in order to examine the stress meter. Its schematic diagram is shown in Figure 9. A borehole was dug inside a tunnel and equipped with casing so that it could be sealed. The 


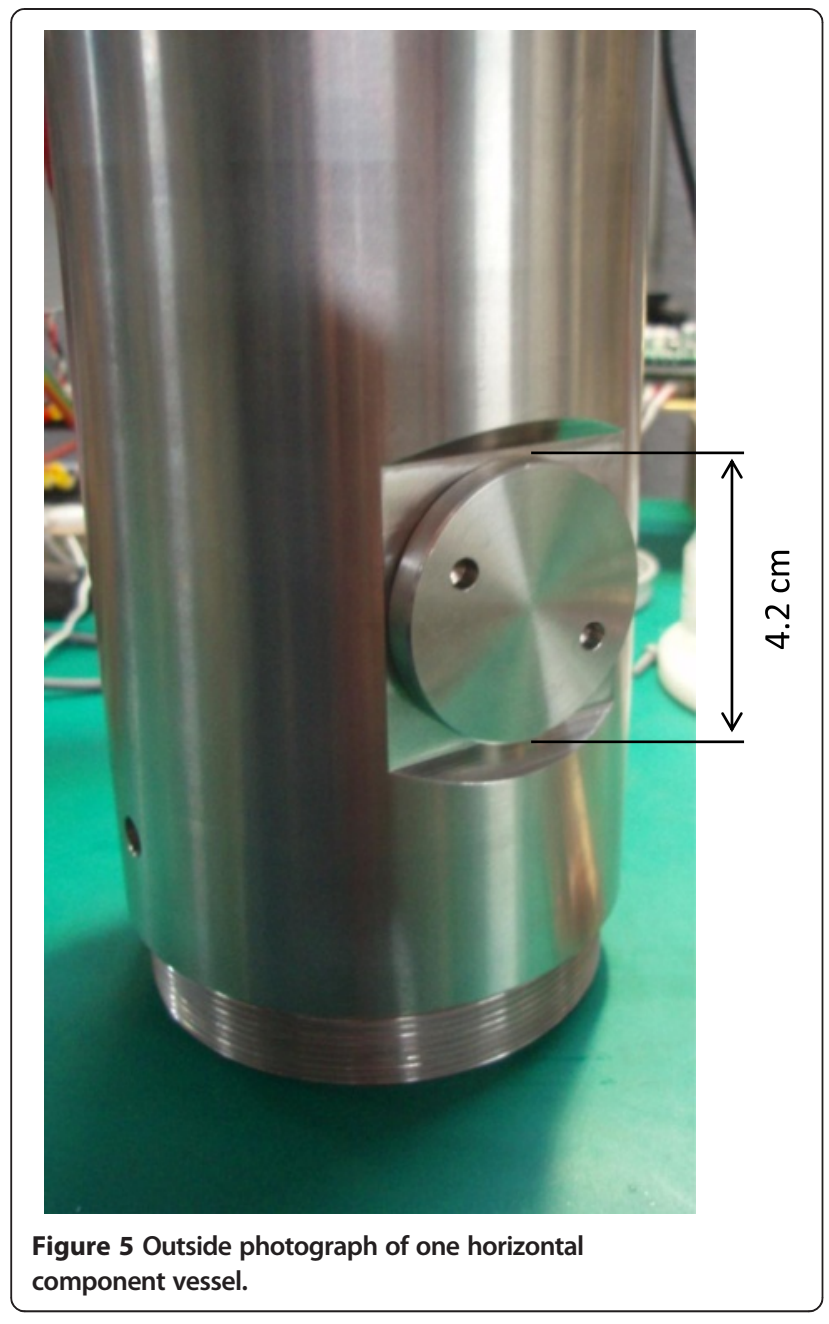

observation device was inserted into the casing, which was then filled with water with the signal cable drawn outward. The observation device was completely sealed, and the water pressure inside the casing could be varied using the water pump. The examination device was used to study the relationship between the given water pressure and the output voltage. Figure 9 shows the setup for the method used to calibrate the stress meter. It is common to use water pressure in order to add stress to the instrument because both stress and water pressure have the same dimension, force/(unit area), and water is manageable for adding pressure (stress). The stress detection part of the stress meter is comprised of two pressure-receiving surfaces, a frame, a magnifying device, and a displacement sensor (pressure-receiving system), as explained in the 'Principle and design of the stress meter' subsection. Thus, the applied water pressure is converted into voltage by displacement. Strictly speaking, a stress meter detects displacement due to pressures (stresses) and displacements (strains) applied only to the pressure-receiving surfaces. In this way, we can measure

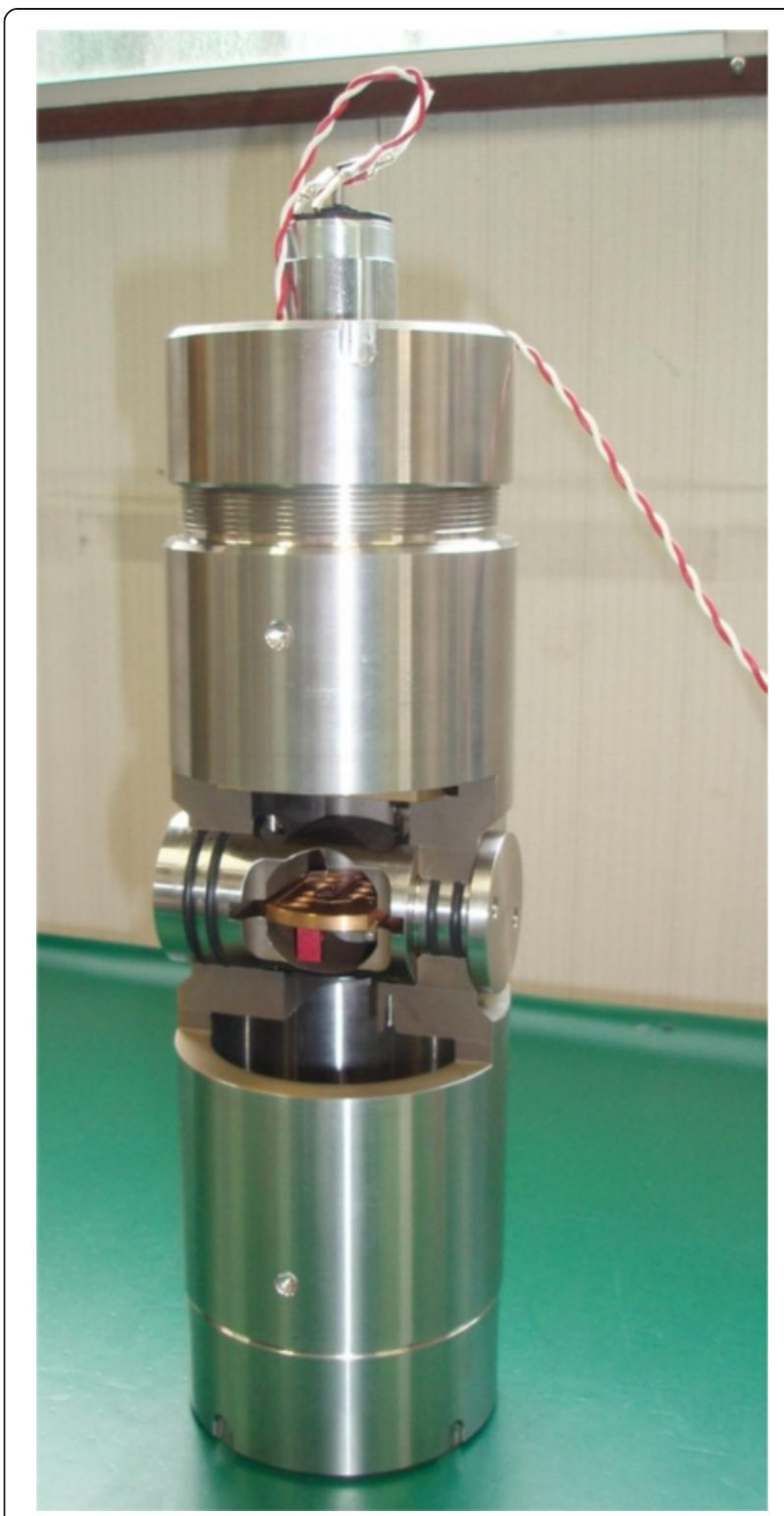

Figure 6 One horizontal component vessel where the stress sensing unit is installed. Half of the vessel is removed to show the installation of the sensing unit.

both rock stress and rock strain changes. Once the relationship between applied pressure and output voltage is deduced, the stress sensitivity of the stress meter can also be deduced. The stress meter to be installed in deep boreholes is usually designed to withstand pressure of about $15 \mathrm{MPa}$ (equivalent to a water column of $1,500 \mathrm{~m}$ ). Figure 10 shows an example of a test result. Only the results for the low-sensitivity vertical component of the stress meter are shown. A linear relationship with output voltage was measured for the stress range from $0 \mathrm{MPa}$ to about $14 \mathrm{MPa}$. Similar results were obtained for the horizontal components. The stress sensitivity examination 


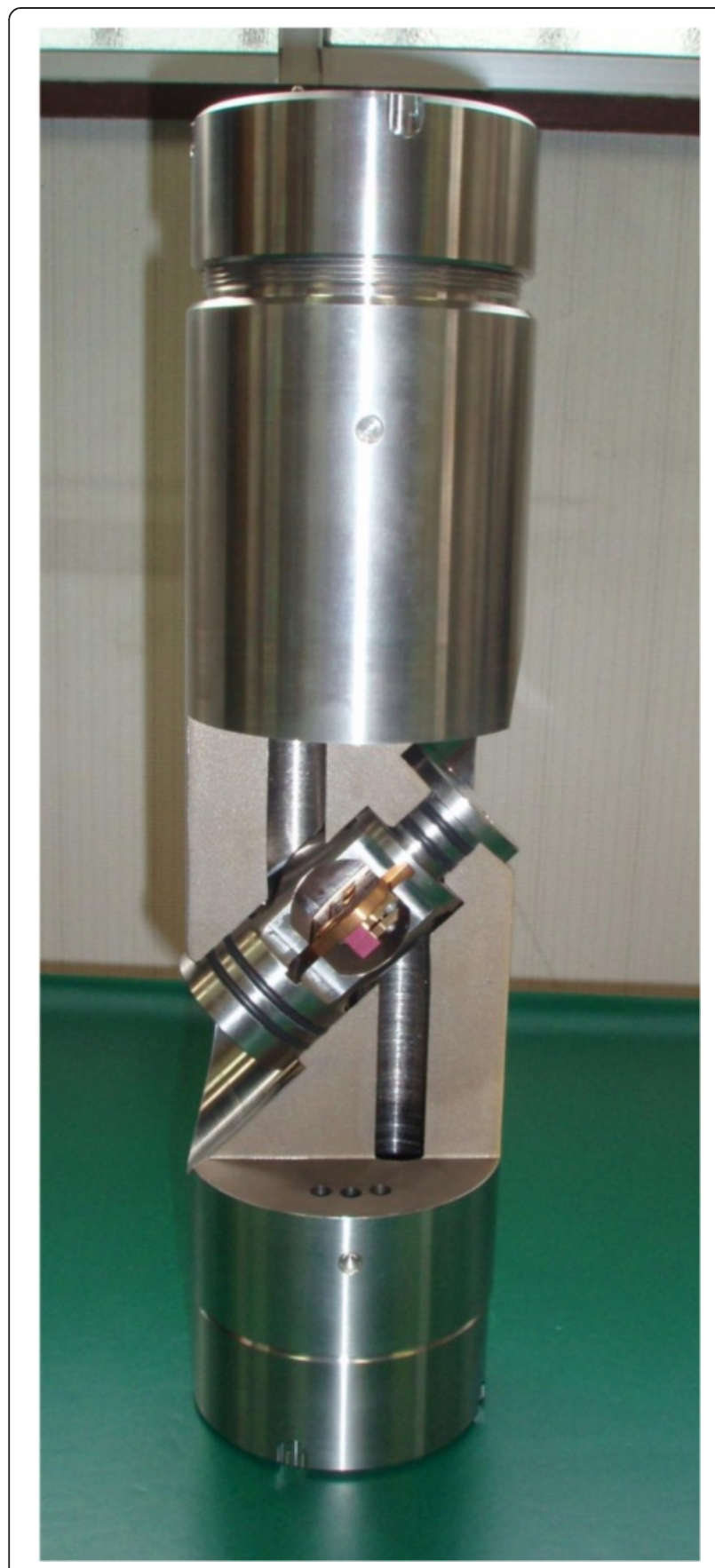

Figure 7 One inclined component vessel where the stress sensing unit is installed. Half of the vessel is removed to show the installation of the sensing unit.

results for the stress meter components incorporated in the comprehensive observation device installed at the TOS borehole station are provided in Table 1 . Based on these examination results, it is possible to observe the stress value and its variation over time at the point of stress meter installation.

\section{Examination of strain meter sensitivity}

Strain examination can be executed by applying displacement to the pressure-receiving surface and measuring the output voltage. Figure 11 shows one case example of such an examination. Only the results for the low-sensitivity vertical component of the stress meter are presented. The output voltage was about $0.1 \mathrm{~V}$ when the strain was about $4 \times 10^{-7}$ with measuring ranges from $10^{-10}$ to $10^{-4}$ strain. A nearly linear relationship occurred. The full strain sensitivity examination results for the TOS borehole station are also shown in Table 1.

\section{Results and discussion}

\section{Stress seismograms of the 11 March 2011 Tohoku earthquake ( $M$ 9.0) and stress tidal records}

The stress meter installed at the bottom of the deep borehole accumulated favorable results. Tidal fluctuations of stress were recorded as long-term changes; seismic stress changes and so forth were recorded as short-term changes. Figure 12 shows the stress seismic motion waveforms recorded by the stress meter in the comprehensive crustal activity observation device at the TOS borehole station (depth of $512 \mathrm{~m}$ ) during the 2011 Tohoku earthquake ( $M$ 9.0), which occurred on 11 March 2011. The epicenter distance was approximately $600 \mathrm{~km}$ from the borehole station. Since the comprehensive borehole crustal activity observation device is also equipped with seismometers, the waveforms observed by the seismometers are also shown for reference. The maximum amplitude of stress seismic waveforms observed at the TOS borehole station was approximately $300 \mathrm{kPa}$.

Figure 13 shows the records observed at the Hiyoshi (HYS) borehole station (depth of $515 \mathrm{~m}$ ) for 10 days. The HYS station $\left(35.421^{\circ} \mathrm{N} ; 137.237^{\circ} \mathrm{E}\right)$ is located in the Gifu Prefecture in central Japan. These data include stress records for four horizontal components, two slant components, and the vertical component. The stress tide values were clearly recorded.

\section{Stress and strain waveforms}

The stress meter can observe both stress and strain, and examinations were conducted on both of these variables as described in the previous section. Figure 14 shows the components of the stress seismic motion waveforms and strain seismic motion waveforms observed at the TOS borehole station during the 2011 Tohoku earthquake (M 9.0) of 11 March 2011. The epicenter distance was approximately $600 \mathrm{~km}$ from the borehole. Because both stress and strain can be observed with the meter, we can obtain a variety of information about the earthquake. This topic will be discussed in more detail at a future opportunity. 


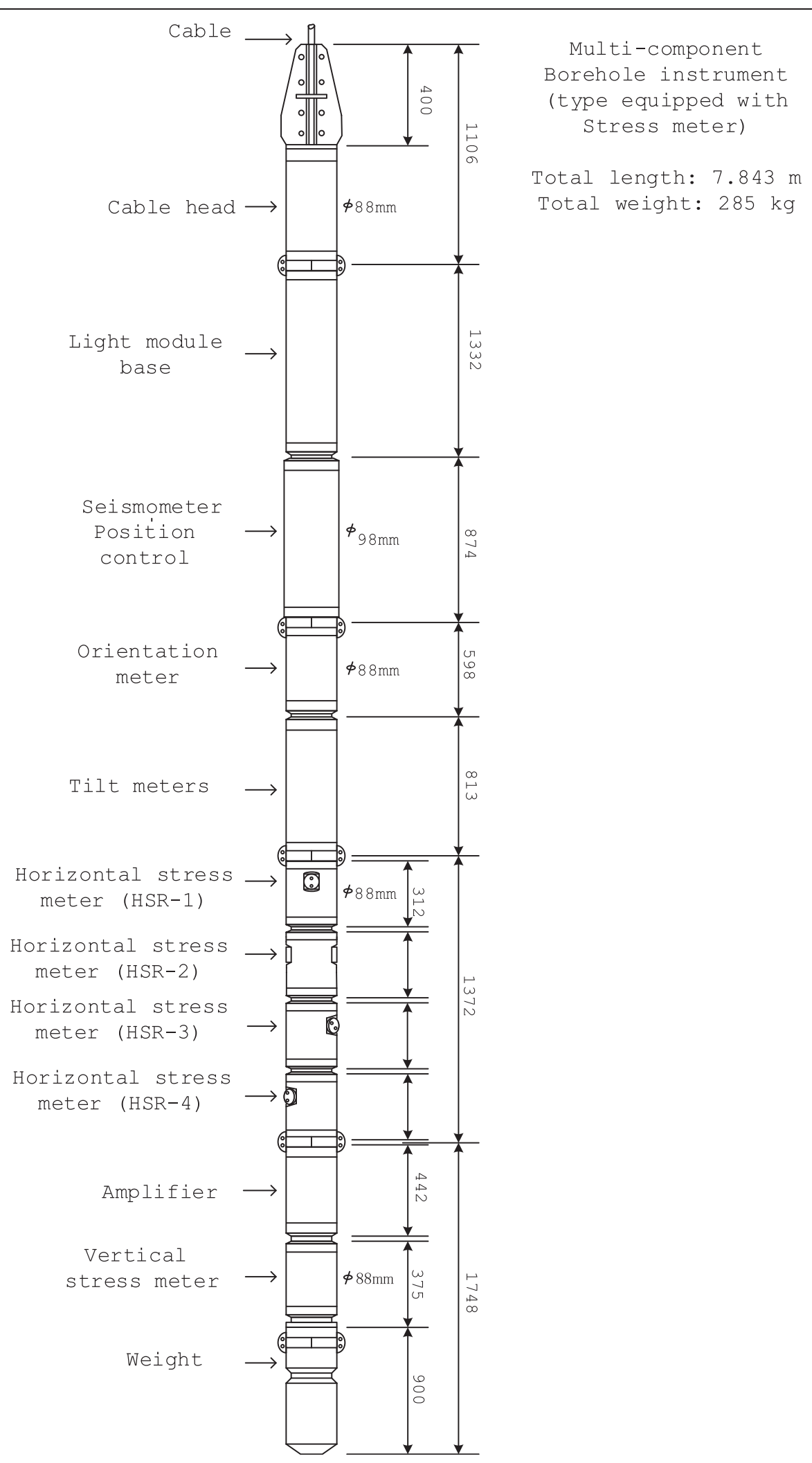

Figure 8 Multi-component borehole instrument. The instrument is equipped with stress meters, being installed in the Toshinomori (TOS) borehole station at a depth of $512 \mathrm{~m}$. HSR, horizontal stress meter. 


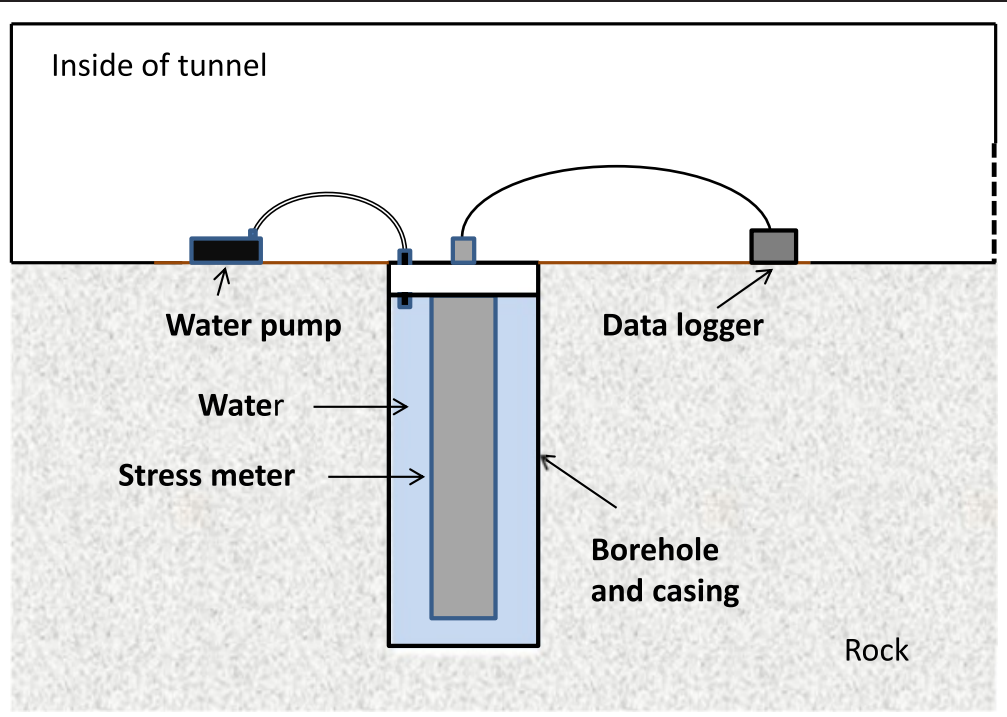

Figure 9 Schematic depiction of the calibration system of the stress meter.

Comparison of stress seismic motion with water pressure seismic motion

Although the sensitivity of the stress meter has been examined, we made a comparison against data observed using a water pressure meter to confirm the reliability. This was done with one of the several borehole stations where stress meters are installed along with water pressure meters (STG200 borehole station; Figure 15). The STG200 station $\left(\mathrm{N} 35.379^{\circ} \mathrm{E} ; 137.240^{\circ} \mathrm{N}\right)$ is located in the Gifu Prefecture in central Japan. Although the arrangement is presented schematically, the bedrock is considered to be a poroelastic body. A borehole with a diameter of approximately $15 \mathrm{~cm}$ and depth of $20 \mathrm{~m}$ was dug inside a tunnel located $200 \mathrm{~m}$ beneath the ground, and the stress meter was installed at the bottom of

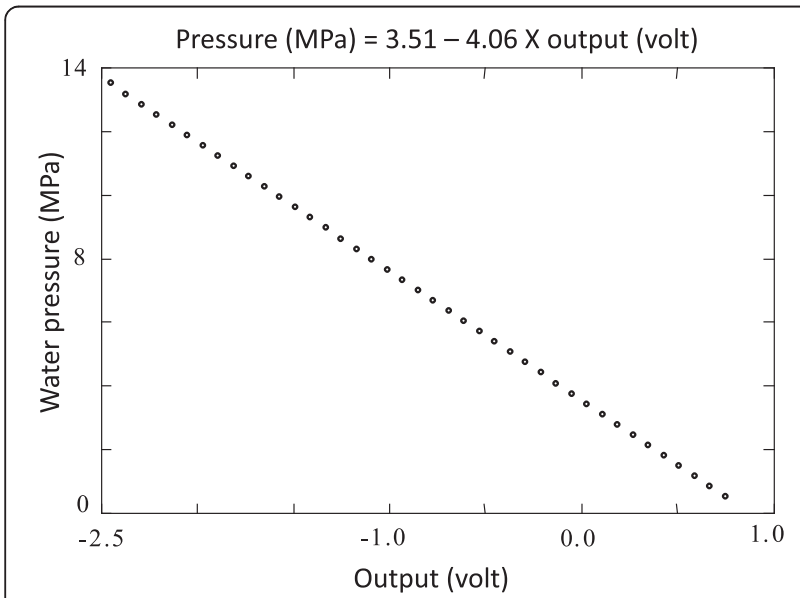

Figure 10 An example calibration showing the relationship between the applied water pressure and output voltage for the stress meter. the borehole and fixed to the surrounding bedrock using expansible grout. The casing in the borehole has a hole in the middle through which water can pass. With this arrangement, the borehole is filled with water and the water starts spilling because of water pressure. A commercial water pressure meter was also installed in the borehole, and the opening was sealed.

The waveforms recorded by the water pressure meter and stress meter in the STG200 borehole station were then compared. Figure 16 shows the stress seismic waveforms and water pressure seismic waveforms for the 2011 Tohoku earthquake ( $M$ 9.0). The red line indicates stress seismic waveforms for the vertical component, and the black line indicates the water pressure seismic waveforms. It is evident from the graph that the stress seismic waveforms and the water pressure seismic waveforms matched very well, including the small changes from the point of wave arrival to the succeeding waves. The good

Table 1 Calibration table for both stress and strain of the meter

\begin{tabular}{|c|c|c|c|c|c|c|}
\hline \multicolumn{7}{|c|}{ Stress $(\mathrm{MPa})=-A \times$ output $(\mathrm{V})+B$} \\
\hline & HSR1 & HSR2 & HSR3 & HSR4 & VSRH & VSRL \\
\hline A & 0.575 & 0.447 & 0.512 & 0.514 & 0.253 & 4.06 \\
\hline $\mathrm{B}$ & 12.9 & 12.9 & 12.7 & 12.9 & 12.9 & 3.51 \\
\hline \multicolumn{7}{|c|}{ Strain $=A \times$ output $(V) \times 10^{-5}+B \times 10^{-7}$} \\
\hline & HSR 1 & HSR2 & HSR3 & HSR4 & VSRH & VSRL \\
\hline A & 2.94 & 2.73 & 2.77 & 2.80 & 3.29 & 32.2 \\
\hline B & 1.51 & -6.4 & 10.1 & 10.3 & 1.41 & 66.1 \\
\hline
\end{tabular}

The meters equipped in multi-component borehole instrument are installed in the Toshinomori (TOS) station at a depth of $512 \mathrm{~m}$. HSR1 to HSR4: horizontal components. VSRH: vertical component with high sensitivity, VSRL: vertical component with low sensitivity. 


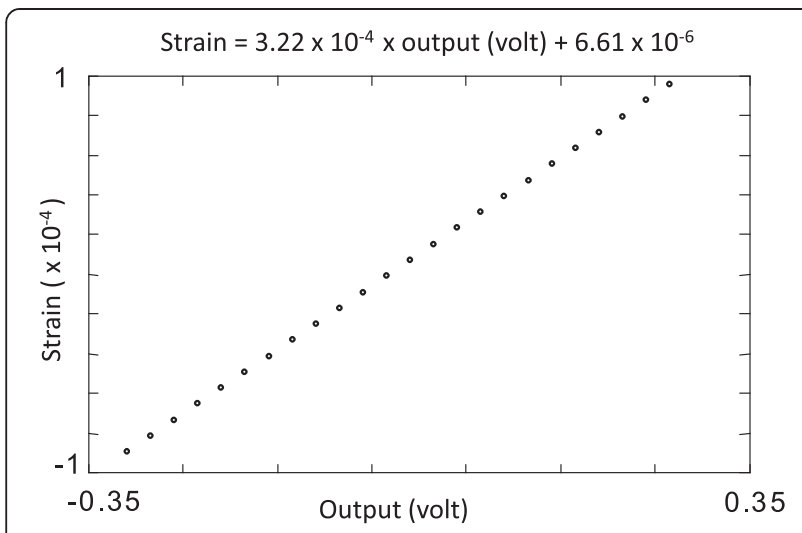

Figure 11 An example calibration showing the relationship between the strain and output voltage for the stress meter.

match of waveforms from the different meters indicates that the signals were recorded properly by both meters. Although both meters recorded the timing of pressure changes precisely, there were some differences in the amplitude of the signals. Specifically, the amplitude for the vertical stress meter was approximately twice as large as that of the water pressure meter. We assumed that this represents the behavior of a poroelastic body in which the water pressure fluctuations that occur are due to fluctuations in bedrock stress.

\section{Stress changes caused by spring water}

Spring water was generated by boring work approximately $300 \mathrm{~m}$ from the water pressure meter (Figure 15). The rate of generation was approximately $200 \mathrm{~L} / \mathrm{min}$. The water pressure and vertical stress component varied radically in concurrence with this, as shown in Figure 17. It is thought that a water vein connects the $300-\mathrm{m}$ point and the observation point and that the changes appeared as a drop in water pressure as a result of the spring water runoff as well as extensional changes in the vertical stress component. The values of both were nearly equal. In this case, it was assumed that the changes in the vertical stress, which were recorded as changes in bedrock stress, were caused by changes in water pressure. The stress meter has been examined for water pressure sensitivity, as described previously. The fact that the vertical stress component reflected the same values as the water pressure changes shows that the sensitivity examination was conducted properly for the stress meter and that the changes in bedrock stress were faithfully recorded without the effects of boring. Many such observation examples exist, and these all indicate the same result.

Case examples of a comparison between vertical stress seismic motion and water pressure seismic motion as well as concurrent changes with spring water were shown above. The structure of the meter is identical for both the horizontal stress component and the vertical stress component. Based on these results, it can be surmised that the stress meter properly records changes in stress. The reliability of the horizontal stress component can be examined by calculating the invariant for stress. This matter will be discussed in a different report.

\section{Considerations on the reliability of the developed stress meter}

Here, we briefly describe the installation method of the borehole stress meter before discussing its reliability. The instrument is fixed inside a deep borehole with expansible grout. A rock sample of predetermined depth is extracted by overcoring before installing the instrument. Then, the borehole is examined to see if there are any cracks or water paths. If there are any, a deeper borehole is dug. Finally, the instrument is installed in uniform bedrock.

We will conduct the following tasks hereafter to investigate the reliability of the stress meter:

1. Check if the observed stress data satisfy the elastic invariants.

2. Perform a comparison between the observed stress tidal variation and theoretical tidal variation.

3. Perform a comparison between the observed stress waveform for the Tohoku earthquake and the theoretical stress waveform.

According to our preliminary investigation, we have obtained good results, and we will soon submit a more comprehensive paper on the reliability of the meter. Here, we just present data on the reliability of the developed stress meter that are clear at the present moment.

Only the pressure-receiving system of the stress meter detects stress and strain. We will now describe the reason why the cylindrical case does not affect the detection. The system is composed of two pressure-receiving surfaces, a frame, a magnifying device, and a displacement sensor. This pressure-receiving system is on the axis orthogonal to the case axis, and it is exposed at the outer wall. The system is thus movable in the axial direction without a mechanical connection as an O-ring is installed in the space next to the case. The device also incorporates a displacement detection sensor to detect stress and strain in the bedrock based on the displacement between the two pressure-receiving surfaces. Therefore, the cylindrical case does not at all affect the detection of stress and strain. The pressure-receiving system only contributes to the detection of stress and strain. The device detects pressures (stresses) and displacements (strains) applied only to the pressure-receiving surfaces.

In conventional strain meters, the overall deformation of the case caused by cracks, water paths, and so on becomes correlated, and it may be difficult to accurately and independently detect strain in the bedrock applied 


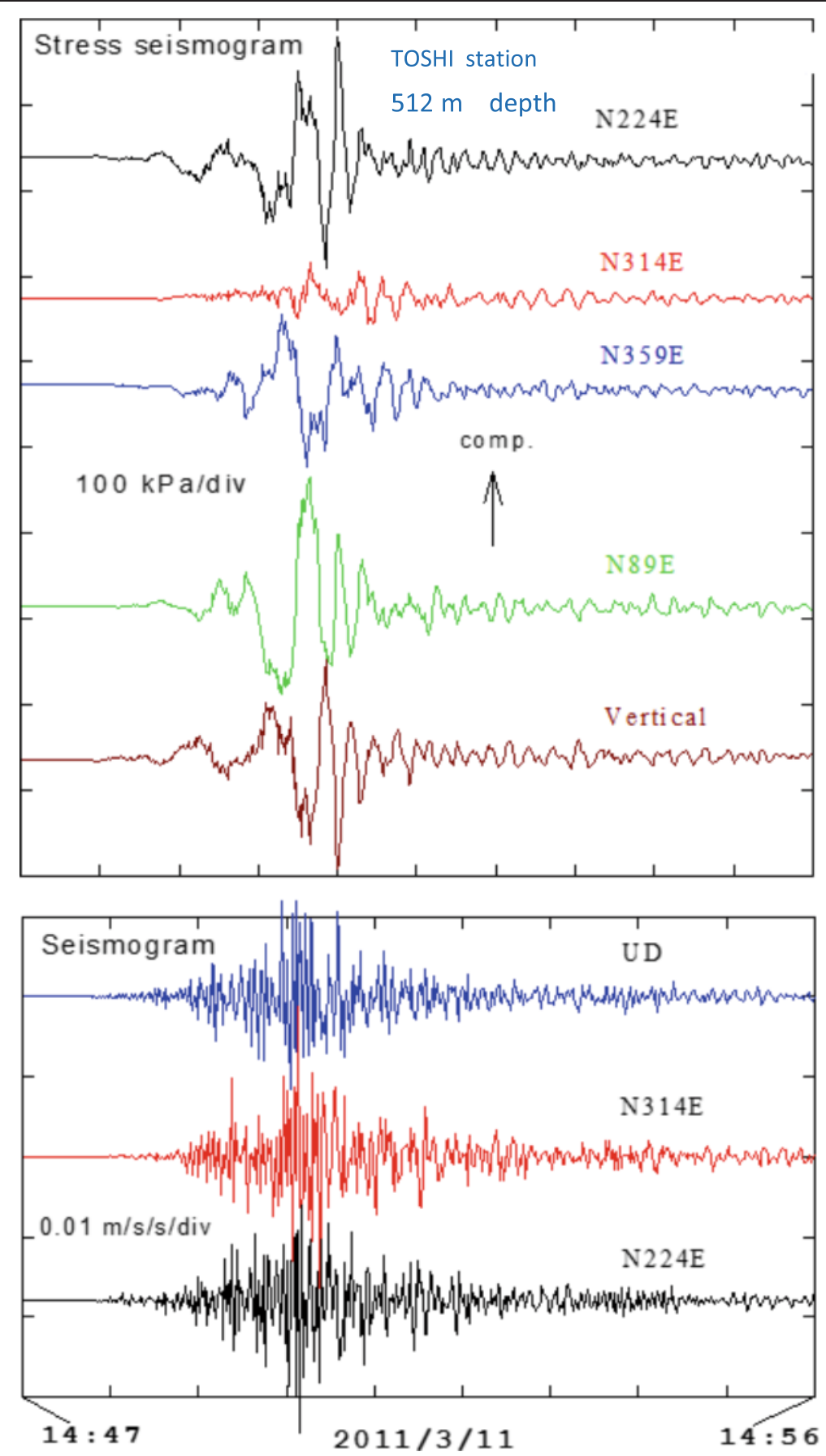

Figure 12 Stress seismogram and normal seismogram observed in the Toshinomori (TOS) borehole station (depth of $512 \mathrm{~m}$ ). During the 2011 Tohoku earthquake (11 March 2011, M 9.0).

against the case from a specified direction. These types of problems have been solved by the stress meter.

As examples of normal stress observations, we present Figures 16 and 17; these data were obtained at borehole station STG200. The area where the station is located is considered to be poroelastic. Figure 15 shows a schematic depiction of the arrangement of the stress meter and water pressure meter, taking into account the poroelastic medium. The waveforms recorded by the water pressure meter and stress meter in borehole station STG200 for the 2011 Tohoku earthquake ( $M$ 9.0) are compared in Figure 16. The vertical component of stress seismic waveforms (red line) and water pressure seismic waveforms (black line) are plotted. It is apparent 


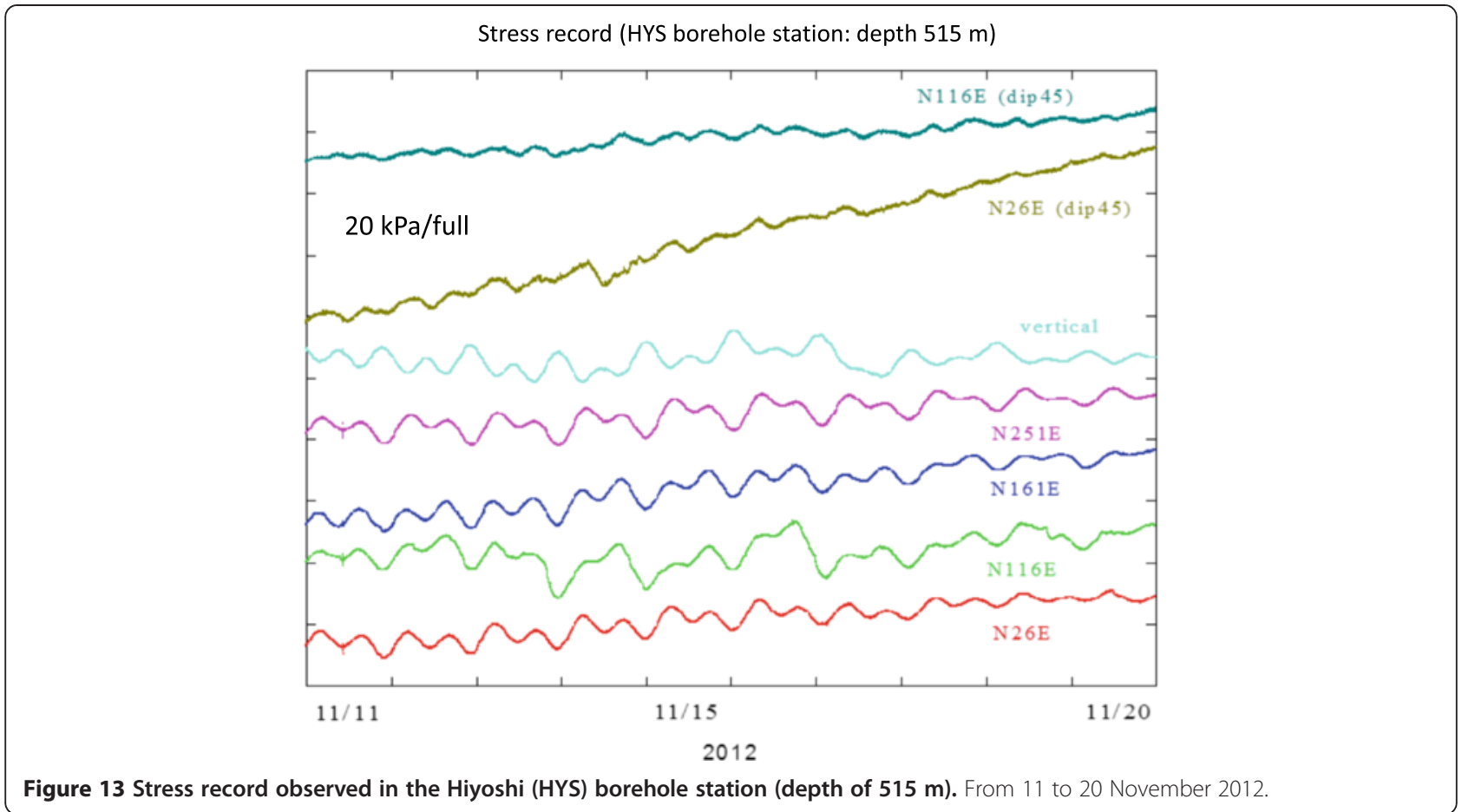

that both waveforms are very similar from the time of wave arrival to the later phases of the waves.

We can interpret this observation as follows. The waves generated by the earthquake arrive at the station and cause stress variation in the elastic part of the poroelastic medium. Stress variation in the elastic part produces a pressure change of pore water in the poroelastic medium, so the water pressure meter records the seismic waveforms. Therefore, the water pressure seismic waveforms are delayed by about $0.35 \mathrm{~s}$ behind the arrival of the stress seismic waveforms.

Figure 17 shows a case where spring water was generated by drilling at a site approximately $300 \mathrm{~m}$ away from the water pressure meter (Figure 15). It is
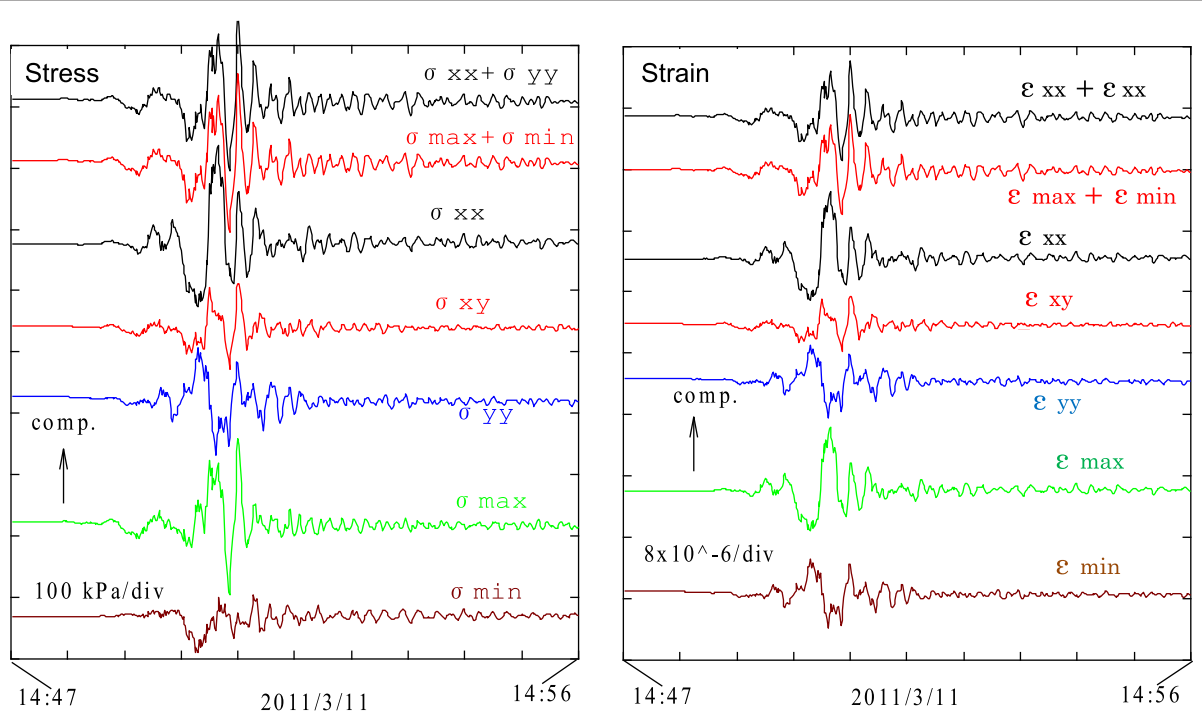

Figure 14 Stress and strain components for the 2011 Tohoku earthquake (March 11, 2011, $\mathbf{M}$ 9.0). Observed by the stress meter at the TOS borehole station. The $X$ axis is in east direction, and the $Y$ axis is in the north direction. Both principal stresses and strains are also shown. 


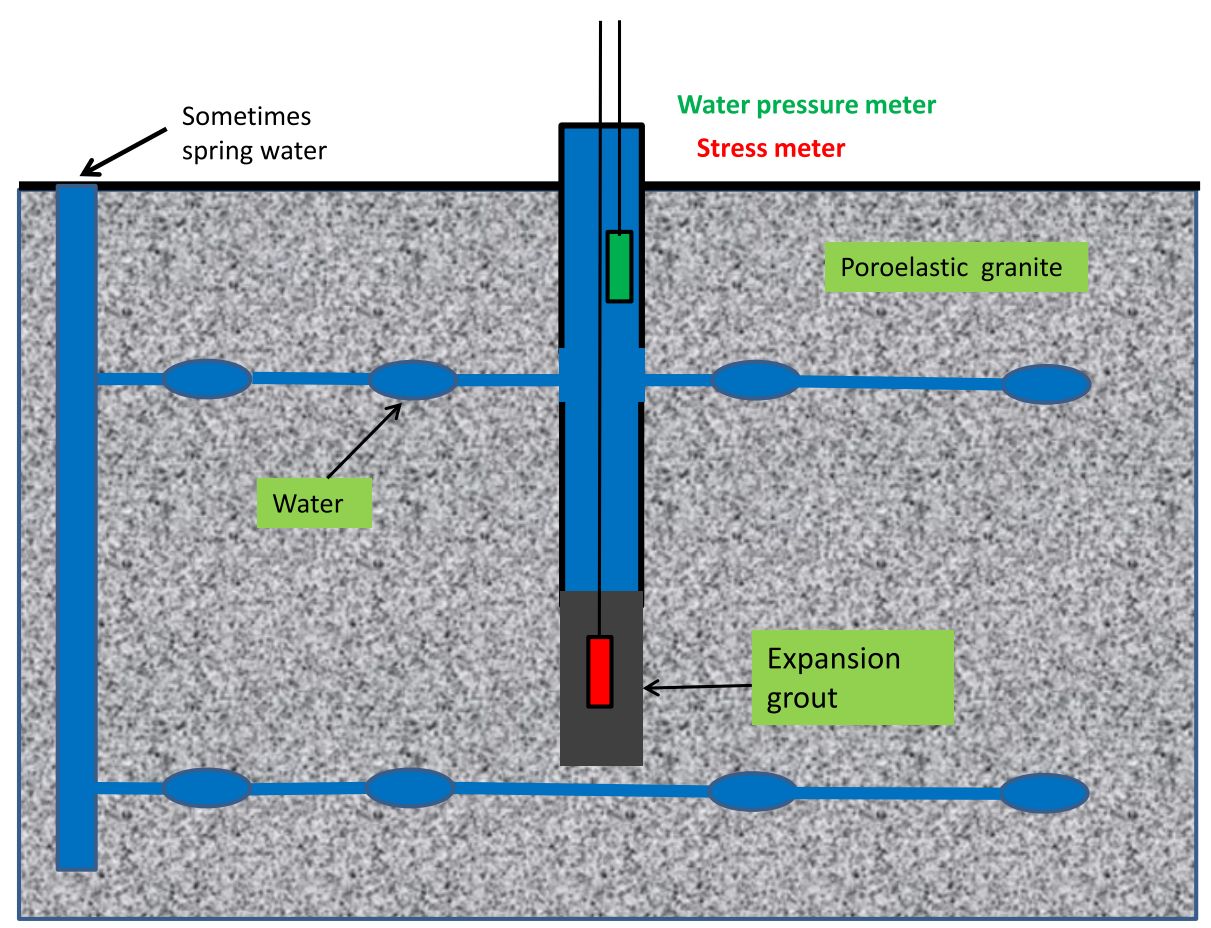

Figure 15 Schematic depiction showing the arrangement of the water pressure meter, stress meter, and aquifer.

thought that a water vein connects the $300-\mathrm{m}$ point and the observation point and that the changes appeared as a drop in pore water pressure as a result of the spring water runoff. A drop in pore water pressure causes extensional changes in the vertical stress in the elastic part of the poroelastic medium, and the stress meter detects extensional changes in vertical stress in the bedrock. Both amplitudes were almost equal. These examples indicate that the stress meter performed well in recording the stress variation.

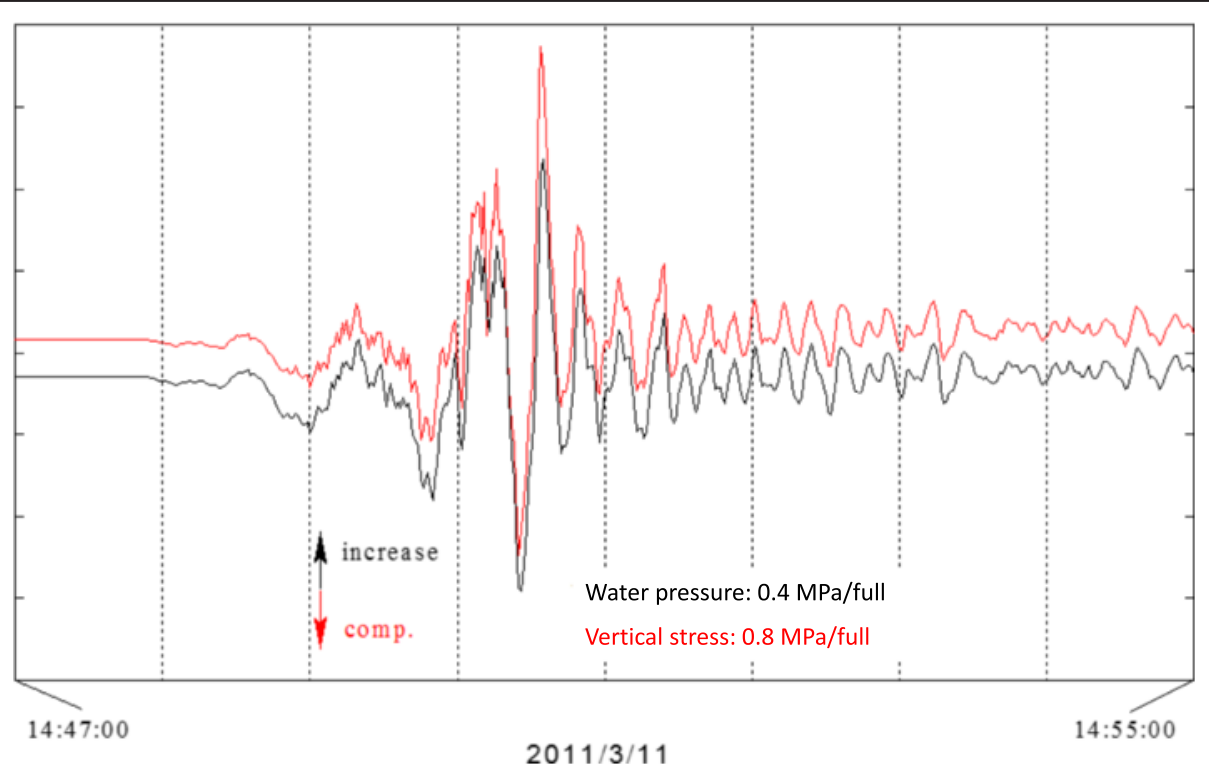

Figure 16 Comparison of records obtained from the vertical component of the stress meter and the water pressure meter. For the 2011 Tohoku earthquake (11 March 2011, M 9.0). 


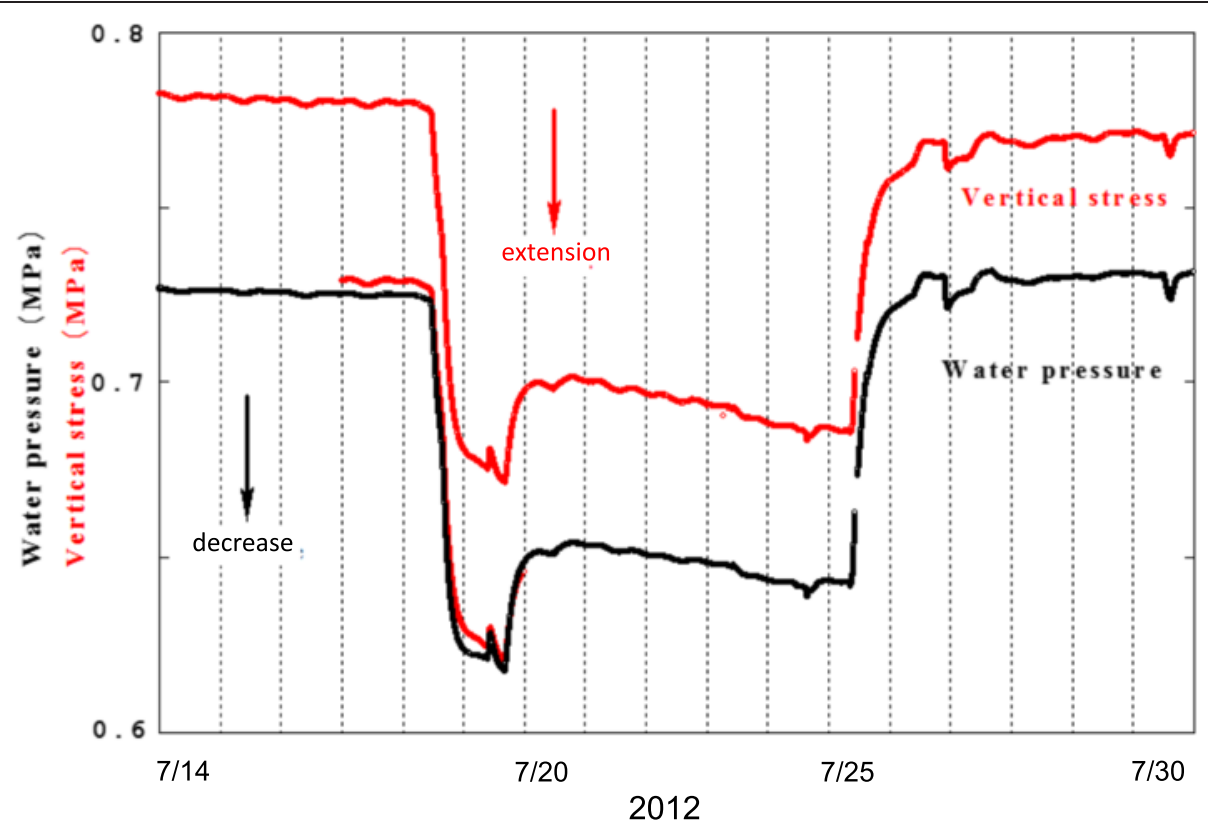

Figure 17 Comparison of records obtained from the stress meter and the water pressure meter. For spring water at a distance of about $300 \mathrm{~m}$ away. A portion of the abrupt change in stress is superimposed on the pressure change curve to show the similarities.

There are two deep borehole stations, TOS and HYS, in addition to borehole station STG200 that are equipped with the stress meter. There are also six borehole stations equipped with conventional strain meters. The tidal strain variations observed at these sites by the stress meters are similar to the tidal variations observed by conventional strain meters. These observations also indicate that the stress meters are performing well.

Based on the discussions above, we believe that the stress meters are performing well in recording both stress and strain variations.

\section{Conclusions}

Although we believe that precursory signs of an earthquake can occur before the event, it is difficult to observe such evidence with precision. This is especially the case when observations are made on the earth's surface, where the precursory signals of earthquakes can be disturbed by artificial noise and other factors. One possible solution to this problem is to make observations with meters installed in the deep bedrock where earthquakes occur.

If continuous observational data can be obtained for stress, we gain access to new information that previously could not be obtained for studying earthquake occurrence and mechanisms and further advances in earthquake research can be expected. However, no instrument capable of continuous observations of stress had been developed. We therefore developed a new borehole stress meter capable of continuous observations of stress change. This stress meter can record continuous observational data for both stress and strain.

This report introduced the design principles for the stress meter and showed the results of sensitivity examinations for stress and strain. At present, such meters are installed and accumulating data in deep boreholes at three observation sites. We also studied the reliability of the stress meter that we manufactured.

Favorable correspondence in the data was found during comparisons of stress meter data and water pressure meter data. As a case example, stress seismic waveforms generated by the 2011 Tohoku earthquake were presented. The results showed that the stress meter that we have developed delivers data with sufficient precision and reliability.

\section{Competing interests}

The authors declare that they have no competing interests.

\section{Authors' contributions}

$\mathrm{HI}$ designed this study, analyzed the data, and wrote the manuscript. YA worked on the installation and maintenance of the instruments and helped with the interpretation of the data. Both authors read and approved the final manuscript.

\section{Acknowledgements}

The authors thank Mr. Hideo Sugaya of Techno Sugaya Co., Ltd. for discussions and comments in regard to developing the stress meter. We also thank Mr. Shigeo Matsumoto for providing useful suggestions throughout the development and installation process. Lastly, we are grateful to the MACOME Corporation, Sumiko Resources Exploration Development Co. Ltd., and System Giden Co. Ltd. for their cooperation and support. 
Received: 21 August 2014 Accepted: 26 January 2015

Published online: 24 February 2015

\section{References}

Asai Y, Okubo M, Ishii H, Aoki H, Yamauchi T, Kitagawa Y, Koizumi N (2005) Co-seismic strain-steps associated with the 2004 off the Kii peninsula earthquakes - observed with Ishii-type borehole strainmeters and quartz-tube extensometers. Earth Planets Space 57:309-314

Gladwin MT (1984) High-precision multicomponent borehole deformation monitoring. Rev Sci Instrum 55:2011-2016

lio Y (2009) Why inland earthquakes happen? Kinmirai company, Nagoya, Japan, pp 1-174 (in Japanese)

Ishii H, Asai Y (2011) Development of borehole stress meter and observed stress data, Poster presentation at: European Geosciences Union, General Assembly, Wien, Austria

Ishii H, Yamauchi T, Kusumoto F (1997a) Development of high sensitivity borehole strain meters and application for rock mechanics and earthquake prediction study. In: Rock Stress. Balkema, The Netherlands, pp 253-258

Ishii H, Chen G, Ohnishi Y (1997b) Estimation of far-field stresses from borehole strain meter observations. In: Rock Stress. Balkema, The Netherlands, pp 259-264

Ishii H, Nakao S, Matsumoto S, Hirata Y (1998) Precursory crustal movements observed by multi-component borehole instrument in the case of earthquake swarms off east coast of Izu peninsula. In: Proceedings of Continuous Crustal Movement Observation in Deep Underground., pp 47-51, in Japanese

Ishii H, Yamauchi T, Matsumoto S, Hirata Y, Nakao S (2002) Development of multi-component borehole instrument for earthquake prediction study: some observed example of precursory and co-seismic phenomena relating to earthquake swarms and application of the instrument for rock mechanics. Seismogenic Process Monitoring, Balkema, The Netherlands, pp 365-377

Itaba S, Koizumi N, Matsumoto N, Ohtani R (2010) Continuous observation of groundwater and crustal deformation for forecasting Tonankai and Nankai earthquakes in Japan. Pure Appl Geophys 167:1105-1114, Doi:10.1007/s00024-010-0095-z

Itaba S, Matsumoto N, Kitagawa Y, Koizumi N (2011) Strain changes after the 2011 off the Pacific coast of Tohoku earthquake (Mw 9.0) observed by a distant borehole strainmeter array. Poster presented at the 2011 European geosciences union, general assembly, Wien, Austria, 8 April 2011

Nishimura T, Munekane H, Yarai H (2011) The 2011 off the Pacific coast of Tohoku Earthquake and its aftershocks observed by GEONET. Earth Planets Space 63:631-636

Okada Y, Yamamoto E, Ohkubo T (2000) Coswarm and preswarm crustal deformation in the eastern Izu Peninsula, central Japan. J Geophys Res 105:681-692

Okubo M, Asai Y, Ishii H (2004) The seismological and geodetical roles of strain seismogram suggested from the 2004 off the Kii peninsula earthquakes. Earth Planets Space 57:303-308

Okubo M, Asai Y, Ishii H, Aoki H (2005) Thousand kilometers breaking of the 2004 Sumatra earthquake proved by dynamic strain analysis. Rep Tono Res Institute of Earthquake Sci 16:81-83

Sacks IS, Suyehiro S, Evertson DW, Yamagishi Y (1971) Sacks-Evertson strain meter, its installation in Japan and some preliminary information concerning strain steps. Papers Meteoro Geophys 22:195-208

Sakata S (1981) On the concept of some newly-invented borehole three-component strainmeters. Rep Nat Res Center Disaster Prev 25:95-126 (in Japanese with English abstract)

Sano O (2005) Measurement methods of contemporary stress field and rock mechanics. J Geogr 114(6):1003-1021 (in Japanese with English abstract)

Yoshida Y, Ueno H, Muto D, Aoki S (2011) Source process of the 2011 off the Pacific coast of Tohoku Earthquake with the combination of teleseismic and strong motion data. Earth Planets Space 63:565-569

\section{Submit your manuscript to a SpringerOpen ${ }^{\circ}$ journal and benefit from:}

- Convenient online submission

- Rigorous peer review

- Immediate publication on acceptance

- Open access: articles freely available online

- High visibility within the field

- Retaining the copyright to your article

Submit your next manuscript at $>$ springeropen.com 